\title{
Numerical Analysis of Two-Arm Spiral Antennas Printed on a Finite-Size Dielectric Substrate
}

\author{
Hisamatsu Nakano, Fellow, IEEE, Hajime Yasui, and Junji Yamauchi, Member, IEEE
}

\begin{abstract}
A two-arm spiral antenna (SA) printed on a dielectric substrate backed by a conducting plane is analyzed under the condition that the dielectric substrate has a finite side length of $L$. The analysis is performed using the finite-difference time-domain (FDTD) method. The computation space for the FDTD method is reduced to one-half of the full computation space by virtue of the antenna arm symmetry with respect to the feed point. The numerical evaluations for the radiation characteristics are briefly described, and the $\mathrm{SA}_{\infty}$ (the spiral antenna with a conducting plane of infinite extent) is analyzed. It is found that the input impedance remains constant with side length $L \geq 0.8$ wavelength in this analysis model. It is also found that as $L$ increases, the axial ratio improves with the gain remaining relatively constant (the gain variation is within $1 \mathrm{~dB}$ ). The $\mathbf{S A}_{\text {finite }}$ (the spiral with a conducting plane having the same finite size as the dielectric substrate) is also analyzed. It is revealed that the effects of $L$ on the axial ratio and gain are relatively large compared with those on the former $\mathrm{SA}_{\infty}$.
\end{abstract}

Index Terms-Finite-difference time-domain (FDTD) method analysis, finite-size dielectric material, spiral antenna.

\section{INTRODUCTION}

$\mathbf{S}$ PIRAL antennas have been analyzed under the condition that the spirals are located in free space or are printed on a dielectric substrate [1]-[9]. The analysis for the printed spirals has been performed with the assumption that the dielectric substrate, backed by a conducting plane, is of infinite extent [2], [5], [9]. Since, in reality, the size of the dielectric substrate is finite, the effects of the finite size on the radiation characteristics need to be investigated.

This paper is a sequel to a previous paper on a two-arm spiral antenna (SA) for circularly polarized wave radiation [10]. The purpose of this paper is to reveal the effects of a finite-size dielectric substrate on the spiral antenna characteristics, including the current distribution, input impedance, radiation pattern, axial ratio, and gain.

First, some techniques for analysis based on the finite-difference time-domain (FDTD) method are described. Making use of the fact that the spiral arms are symmetrical with respect to the feed point, the computation space for the FDTD method is reduced to one-half of the full computation space. A calculation technique for the radiation pattern when the substrate is of infinite extent is also described.

Second, an SA with a finite-size dielectric substrate is analyzed under the condition that the conducting plane is of infinite

Manuscript received March 1, 1999.

The authors are with the College of Engineering, Hosei University, Koganei,

Tokyo, Japan 184-8584 (e-mail: nakano@k.hosei.ac.jp).

Publisher Item Identifier S 0018-926X(02)04569-6.

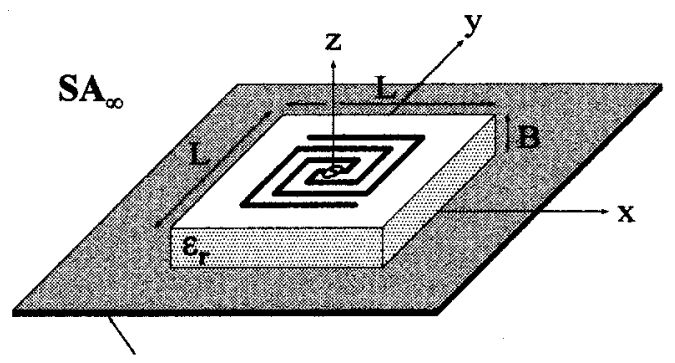

conducting plane of infinte extent

(a)

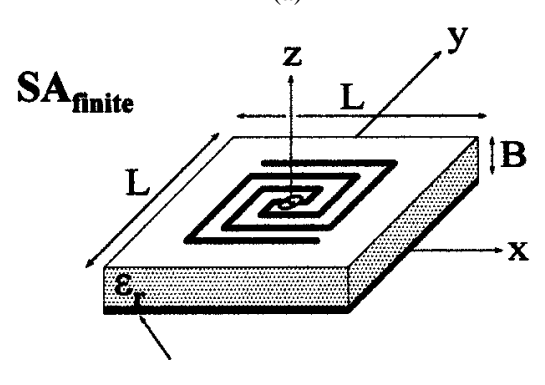

finite-size conducting plane

(b)

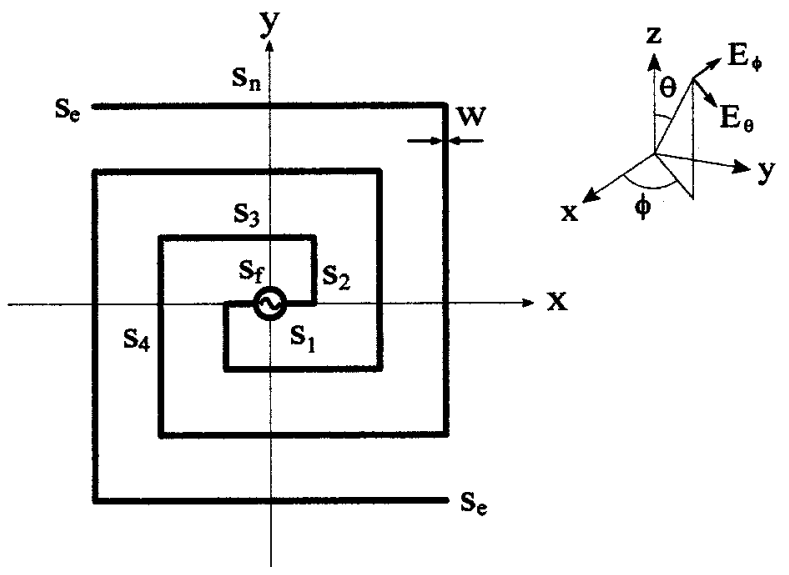

(c)

Fig. 1. Configuration and coordinate system. (a) Spiral antenna with a finite-size dielectric substrate backed by a conducting plane of infinite extent $\left(\mathrm{SA}_{\infty}\right)$, (b) spiral antenna with a finite-size dielectric substrate backed by a finite-size conducting plane $\left(\mathrm{SA}_{\text {finite }}\right)$, and (c) spiral arms: the filaments with width $w$ have lengths $S_{1}, S_{2}, \ldots$, and $S_{n}$.

extent. The SA under this condition, designated as $\mathrm{SA}_{\infty}$, corresponds, for example, to a radiation element mounted on the conducting roof of an automobile. The radiation characteristics are evaluated as a function of the side length of a square dielectric substrate $L$. It is found that as the side length $L$ increases, the 


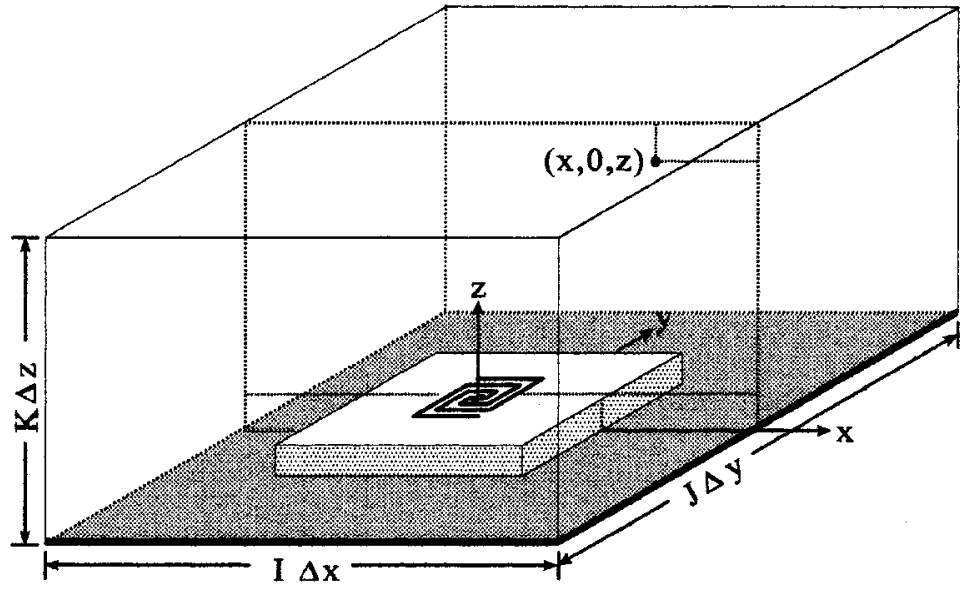

(a)

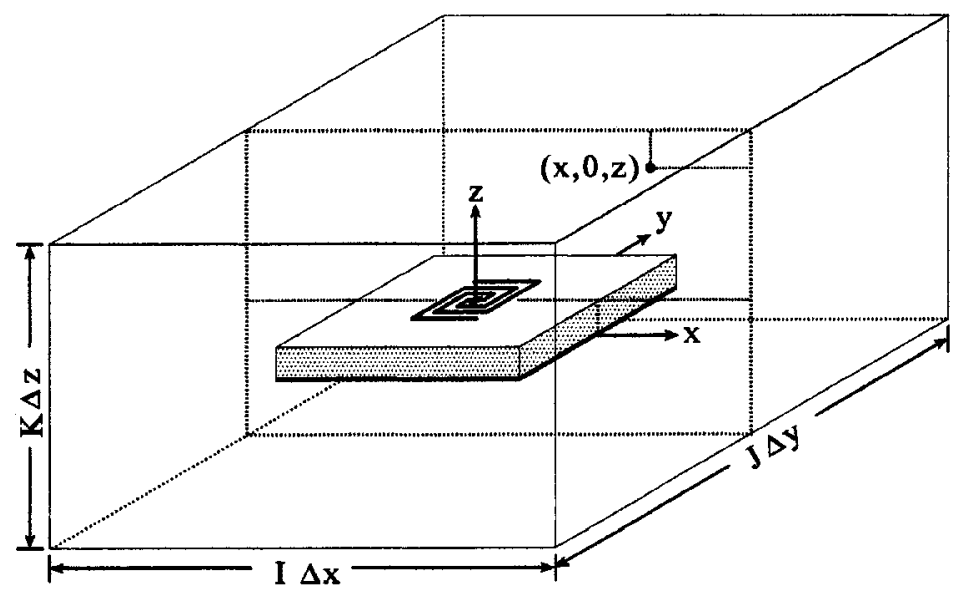

(c)

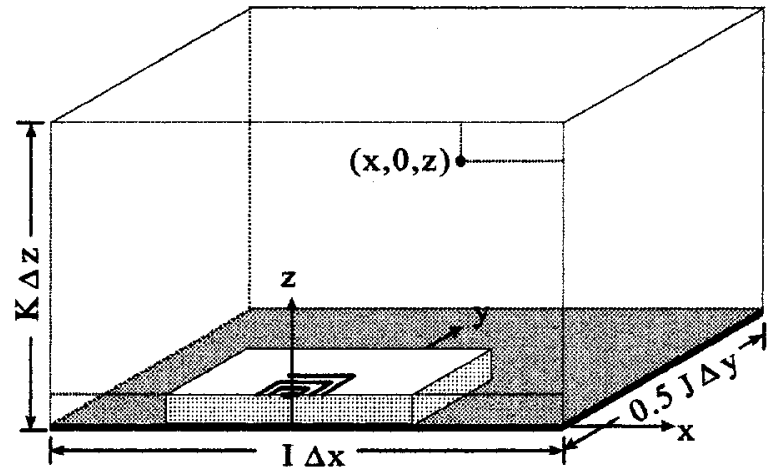

(b)

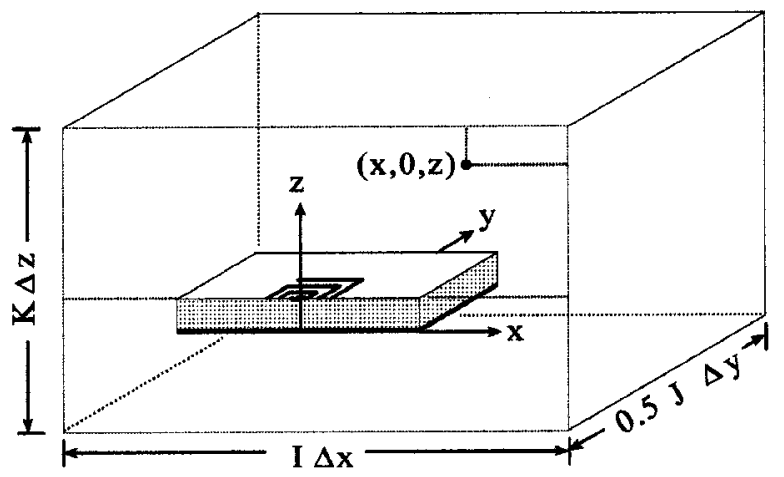

(d)

Fig. 2. Computation space. (a) Full computation space $I \Delta x \times J \Delta y \times K \Delta z$ for $\mathrm{SA}_{\infty}$. (b) Reduced computation space $I \Delta x \times 0.5 J \Delta y \times K \Delta z$ for $\mathrm{SA}$. (c) Full computation space $I \Delta x \times J \Delta y \times K \Delta z$ for $\mathrm{SA}_{\text {finite. }}$. (d) Reduced computation space $I \Delta x \times 0.5 J \Delta y \times K \Delta z$ for $\mathrm{SA}_{\text {finite. }}$.

currents along the spiral arms tend toward a smoothly decaying traveling-wave and the axial ratio improves.

Third, an SA is analyzed under the condition that both the square dielectric substrate and the square conducting plane have the same finite side length $L$ (the $\mathrm{SA}$ is designated as the $\left.\mathrm{SA}_{\text {finite }}\right)$. It is revealed that the side length $L$ for the $\mathrm{SA}_{\text {finite }}$ affects the radiation characteristics more than that for the $\mathrm{SA}_{\infty}$. The behavior in the radiation pattern, axial ratio, and gain are illustrated as a function of the side length $L$. Note that the frequency responses for the $\mathrm{SA}_{\text {finite }}$ are also described in comparison with those for the $\mathrm{SA}_{\infty}$.

\section{CONFIGURATION}

Fig. 1 shows the configuration of a two-arm printed SA on a square dielectric substrate of side length $L$. The dielectric substrate has thickness $B$ and relative permittivity $\varepsilon_{r}$. The dielectric substrate is backed by a conducting plane. The conducting plane in Fig. 1(a) is of infinite extent, while the conducting plane in Fig. 1(b) is square and finite, having the same side length $L$ as the dielectric substrate. These spiral antennas shown in Fig. 1(a) and (b) are designated as $\mathrm{SA}_{\infty}$ and $\mathrm{SA}_{\text {finite }}$, respectively.
The two spiral arms are symmetrically wound with respect to the feed point $s_{f}$, each arm having $N$ filaments, as shown in Fig. 1(c). Throughout this paper, the following configuration parameters are fixed: $B=5 \mathrm{~mm}=0.135 \lambda_{0}, \varepsilon_{r}=2.33, N=7$ (with filament lengths $s_{1}=40 \mathrm{mil}=1.016 \mathrm{~mm}=0.0271 \lambda_{0}$, $s_{2}=1.5 s_{1}, s_{3}=3.5 s_{1}, s_{4}=4.5 s_{1}, s_{5}=6.5 s_{1}, s_{6}=7.5 s_{1}$, and $s_{7}=8 s_{1}$ ), and width of spiral arm $w=0.0108 \lambda_{0}$, where $\lambda_{0}$ is the free-space wavelength at a test frequency of $8 \mathrm{GHz}$. The side length of the dielectric substrate, $L$, is varied in the following analysis.

\section{NUMERICAL ANALYSIS}

The SA is analyzed using the FDTD method [11], [12], in which Yee's algorithm [13] is adopted with Liao's second order absorbing boundary condition [14].

The SA is excited with a delta-gap voltage source, which is expressed with a sine function modulated by a Gaussian function [15]. The symmetry of the antenna arms with respect to the feed point $s_{f}$ leads to the following relationships in the electric 


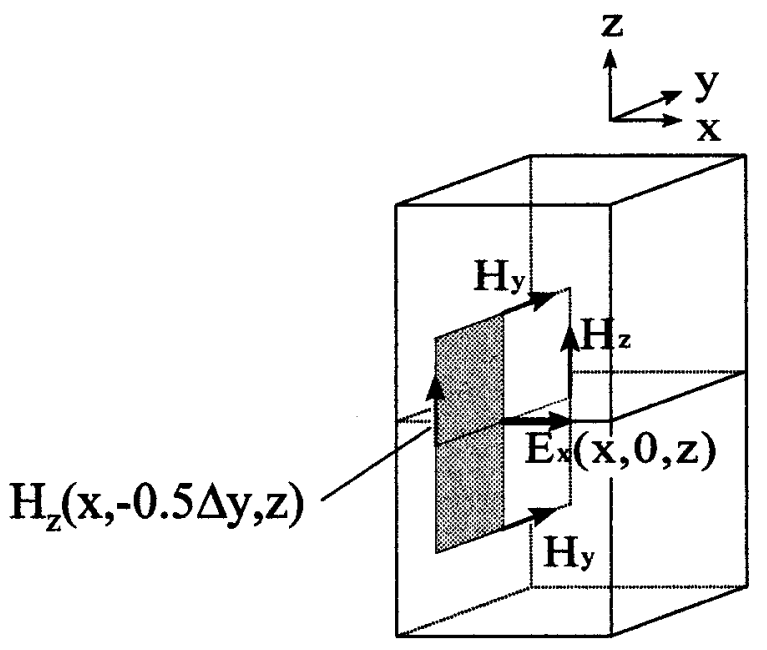

(a)

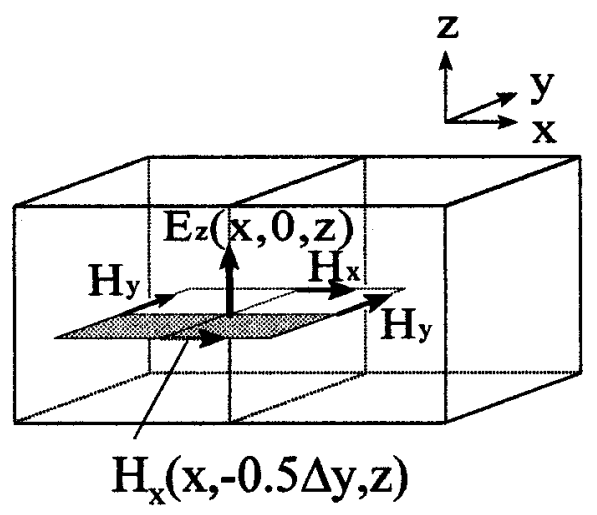

(b)

Fig. 3. Electric fields $E_{x}$ and $E_{z}$ in the $x-z$ plane (computation space boundary).

field components $\left(E_{x}, E_{y}, E_{z}\right)$ and the magnetic field components $\left(H_{x}, H_{y}, H_{z}\right)$ :

$$
\begin{aligned}
& E_{x}(x, y, z)=E_{x}(-x,-y, z) \\
& E_{y}(x, y, z)=E_{y}(-x,-y, z) \\
& E_{z}(x, y, z)=-E_{z}(-x,-y, z) \\
& H_{x}(x, y, z)=H_{x}(-x,-y, z) \\
& H_{y}(x, y, z)=H_{y}(-x,-y, z) \\
& H_{z}(x, y, z)=-H_{z}(-x,-y, z) .
\end{aligned}
$$

Using these symmetry relationships, the computation space shown in Fig. 2(a) and (c) is reduced to one-half of the full computation space, shown in Fig. 2(b) and (d). That is, a computation space of $(I \Delta x, J \Delta y, K \Delta z)$ is reduced to ( $I \Delta x$, $0.5 J \Delta y, K \Delta z)$, where $\Delta x, \Delta y$, and $\Delta z$ are the side lengths of a grid cell in the $x, y$, and $z$ directions, respectively, and $I$, $J$, and $K$ are integers.

The $E_{x}(x, 0, z)$ at the computation space boundary $(x-z$ plane) is determined by using Yee's algorithm with four magnetic fields surrounding the $E_{x}(x, 0, z)$ [see Fig. 3(a)]. Note

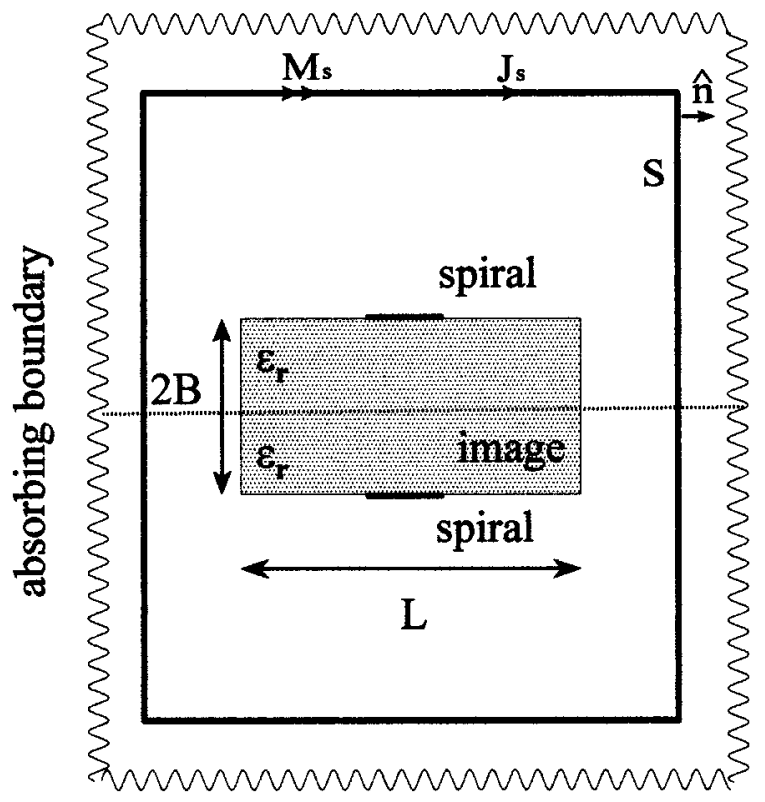

$\mathbf{S A}_{\infty}$

(a)

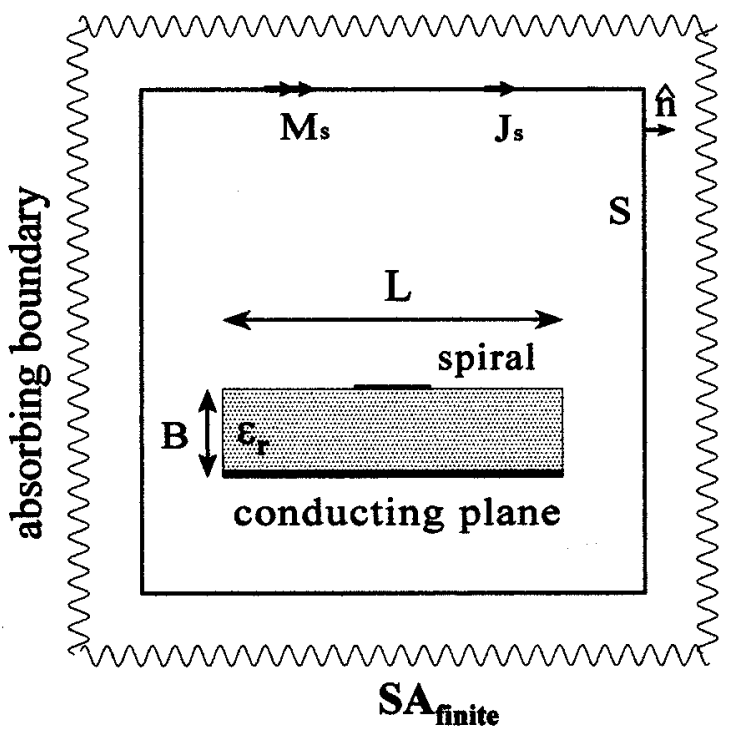

(b)

Fig. 4. Enclosing surface for far-field calculation; $\mathbf{M}_{s}$ and $\mathbf{J}_{s}$ are magnetic and electric currents on the enclosing surface, respectively. (a) $\mathrm{SA}_{\infty}$ : the conducting plane is removed using image theory. (b) $\mathrm{SA}_{\text {finitc }}$.

that one of the four magnetic fields, $H_{z}(x,-0.5 \Delta y, z)$, is located beyond the computation space defined in Fig. 2(b) and (d), and can not be used for the $E_{x}(x, 0, z)$ calculation. However, this $H_{z}(x,-0.5 \Delta y, z)$ is replaced with $H_{z}(-x, 0.5 \Delta y, z)$, which is located within the computation space in Fig. 2(b) and (d), using the symmetry condition (2c)

$$
H_{z}(x,-0.5 \Delta y, z)=-H_{z}(-x, 0.5 \Delta y, z) .
$$

Similarly, the $E_{z}(x, 0, z)$ at the computation space boundary is determined by using Yee's algorithm with $H_{x}(x, \pm 0.5 \Delta y, z)$ and $H_{y}(x, \pm 0.5 \Delta x, 0, z)$ [see Fig. 3(b)]. Using the symmetry 

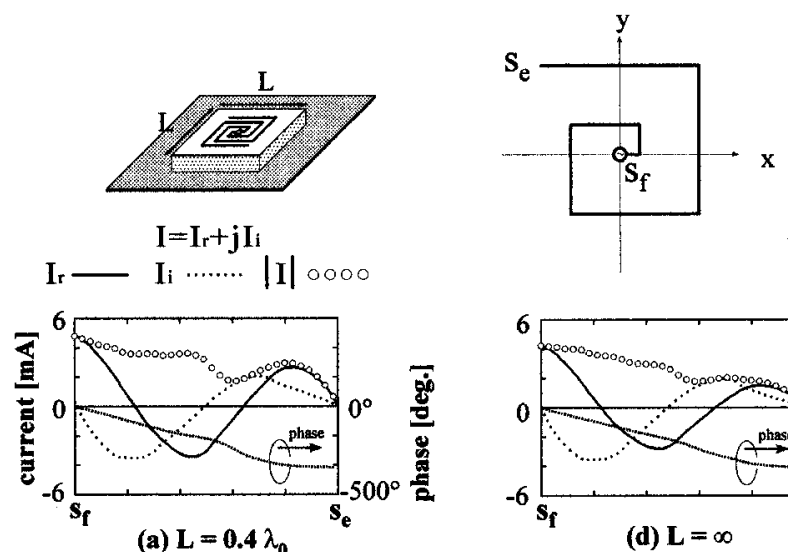

(a) $\mathrm{L}=0.4 \lambda_{0}$

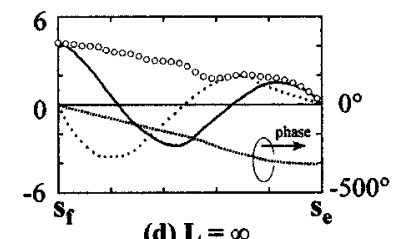

(d) $\mathbf{L}=\infty$

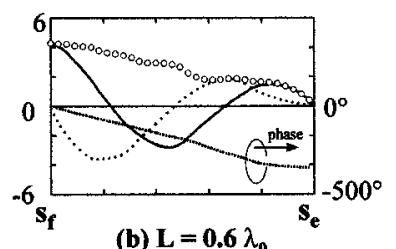

(b) $\mathbf{L}=0.6 \lambda$

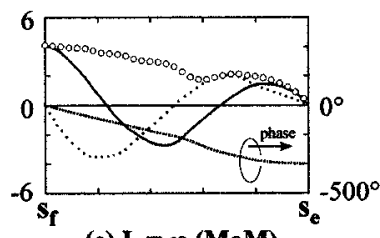

(e) $\mathbf{L}=\infty$ (MoM)

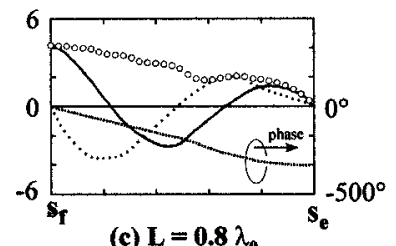

(c) $L=0.8 \lambda_{0}$

Fig. 5. Current distribution $I=I_{r}+j I_{i}$ of $\mathrm{SA}_{\infty}$ as a function of side length $L$. (a)-(d) results calculated using the FDTD method and (e) result calculated using the MoM. In (d), the FDTD method solution for $L=\infty$ agrees well with (e) the MoM solution.

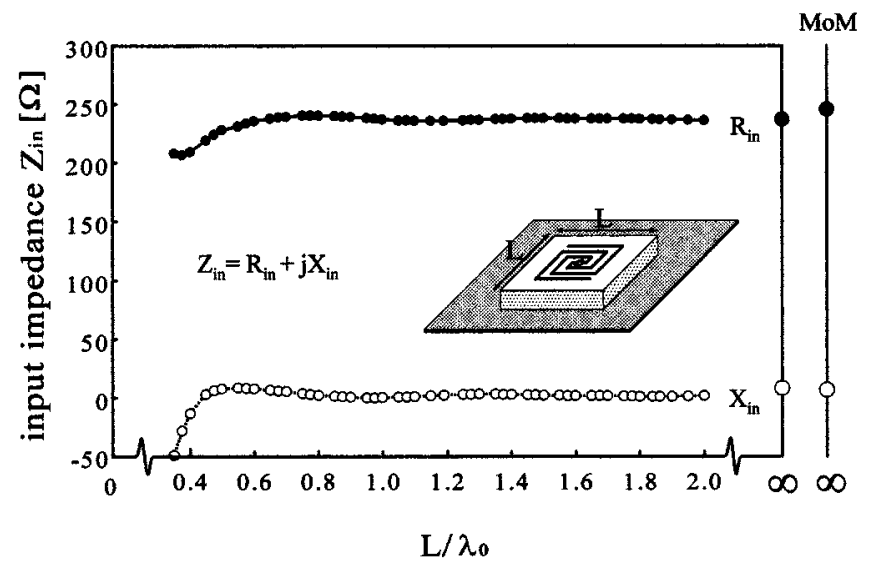

Fig. 6. Input impedance $Z_{i n}=R_{i n}+j X_{i n}$ of $\mathrm{SA}_{\infty}$ as a function of normalized side length $\left(L / \lambda_{0}\right)$. Results calculated using MoM are also presented for $L=\infty$.

condition (2-a), $H_{x}(x,-0.5 \Delta y, z)$, located beyond the computation space defined in Fig. 2(b) and (d), is replaced with $H_{x}(-x, 0.5 \Delta y, z)$ located within the computation space

$$
H_{x}(x,-0.5 \Delta y, z)=H_{x}(-x, 0.5 \Delta y, z) .
$$

The current distribution along the spiral arm $I\left(s^{\prime}\right)\left(s^{\prime}\right.$ is the distance measured along the spiral arm from the feed point $s_{f}$ to

the current source point) is obtained by Fourier-transforming the time-domain current determined by integrating the time-domain magnetic field around the spiral arm (Ampere's circuital law). Using the Fourier-transformed current $I(0)$ and voltage $V(0)$ at the input terminals, the input impedance is evaluated as $Z_{\text {in }}=$ $V(0) / I(0)$.

The far-field $\mathbf{E}_{f a r}=E_{\theta} \hat{\theta}+E_{\phi} \hat{\phi}(\hat{\theta}$ and $\hat{\phi}$ are unit vectors for the spherical coordinates $\theta$ and $\phi$, respectively) is calculated on the basis of the equivalence principle, which uses the electric current $\mathbf{J}_{s}=\hat{n} \times \mathbf{H}$ and magnetic current $\mathbf{M}_{s}=\mathbf{E} \times \hat{n}$ on the surface $S$ that encloses the antenna (see Fig. 4) [12], where $\mathbf{H}$ and $\mathbf{E}$ are, respectively, the magnetic and electric fields on the surface $S$, and $\hat{n}$ is the outward unit vector normal to the surface $S$.

The current $I\left(s^{\prime}\right)$ can be obtained on the basis of the FDTD method even when the side lengths of the square dielectric and conducting plane are infinite $(L=\infty)$. However, the far-field $\mathbf{E}_{\text {far }}$ for $L=\infty$ cannot be obtained from the FDTD method, because the antenna cannot be enclosed due to the infinite dielectric and conducting planes. For this case, the far-field is formulated using two Sommerfeld-type Green's functions [2], which include the effects of the arm image produced by the conducting plane of infinite extent, together with the current $I\left(s^{\prime}\right)$ obtained by the FDTD method. Adopting the stationary phase method (SPM) [2] simplifies the two Green's functions and leads to the far-field components $(i=\theta, \phi)$

$$
\begin{aligned}
E_{i}=-j 60 k_{0} \frac{e^{-j k_{0} R}}{R} \cos \theta F_{i}(\theta) \int_{-s_{e}}^{+s_{e}} \\
\cdot\left[x^{\prime}\left(s^{\prime}\right) \tau_{i}+y^{\prime}\left(s^{\prime}\right) \gamma_{i}\right] I\left(s^{\prime}\right) e^{j k_{0} \Psi\left(s^{\prime}\right)} d s^{\prime}
\end{aligned}
$$

where

$$
\begin{aligned}
F_{\theta}(\theta) & =\frac{\xi \sin \left(k_{0} B \xi\right)}{\xi \sin \left(k_{0} B \xi\right)-j \varepsilon_{r} \cos \theta \cos \left(k_{0} B \xi\right)} \\
F_{\phi}(\theta) & =\frac{\sin \left(k_{0} B \xi\right)}{\cos \theta \sin \left(k_{0} B \xi\right)-j \xi \cos \left(k_{0} B \xi\right)} \\
\Psi\left(s^{\prime}\right) & =x\left(s^{\prime}\right) \sin \theta \cos \phi+y\left(s^{\prime}\right) \sin \theta \sin \phi+B \cos \theta .
\end{aligned}
$$

The notation in the previous equations is as follows: $R$ is the distance between the spherical coordinate origin and the observation point; $k_{0}$ is the wave number in free space; $x\left(s^{\prime}\right)$ and $y\left(s^{\prime}\right)$ are the rectangular coordinates specifying the position of a point defined by $s^{\prime} ; x^{\prime}\left(s^{\prime}\right)$ and $y^{\prime}\left(s^{\prime}\right)$ are derivatives with respect to $s^{\prime} ; \xi=\left(\varepsilon_{r}-\sin ^{2} \theta\right)^{1 / 2}, \tau_{\theta}=\cos \phi, \gamma_{\theta}=\sin \phi, \tau_{\phi}=-\sin \phi$, and $\gamma_{\phi}=\cos \phi$. Note that (5) is derived under the condition that the current flows along a wire whose radius $\rho$ is small enough for the thin wire approximation to be applied. The wire radius $\rho$ is approximated by the strip width $w$ as $\rho=w / 4$.

The spirals shown in Fig. 1 are expected to radiate a right-hand circularly polarized (RHCP) wave due to the winding sense of the spiral arm. The gain for a RHCP wave in the direction specified by the spherical coordinates $(R, \theta, \phi)$, $G_{R}$, is expressed as

$$
G_{R}=\frac{\left|E_{R}\right|^{2} R^{2}}{30 P_{\text {in }}}
$$




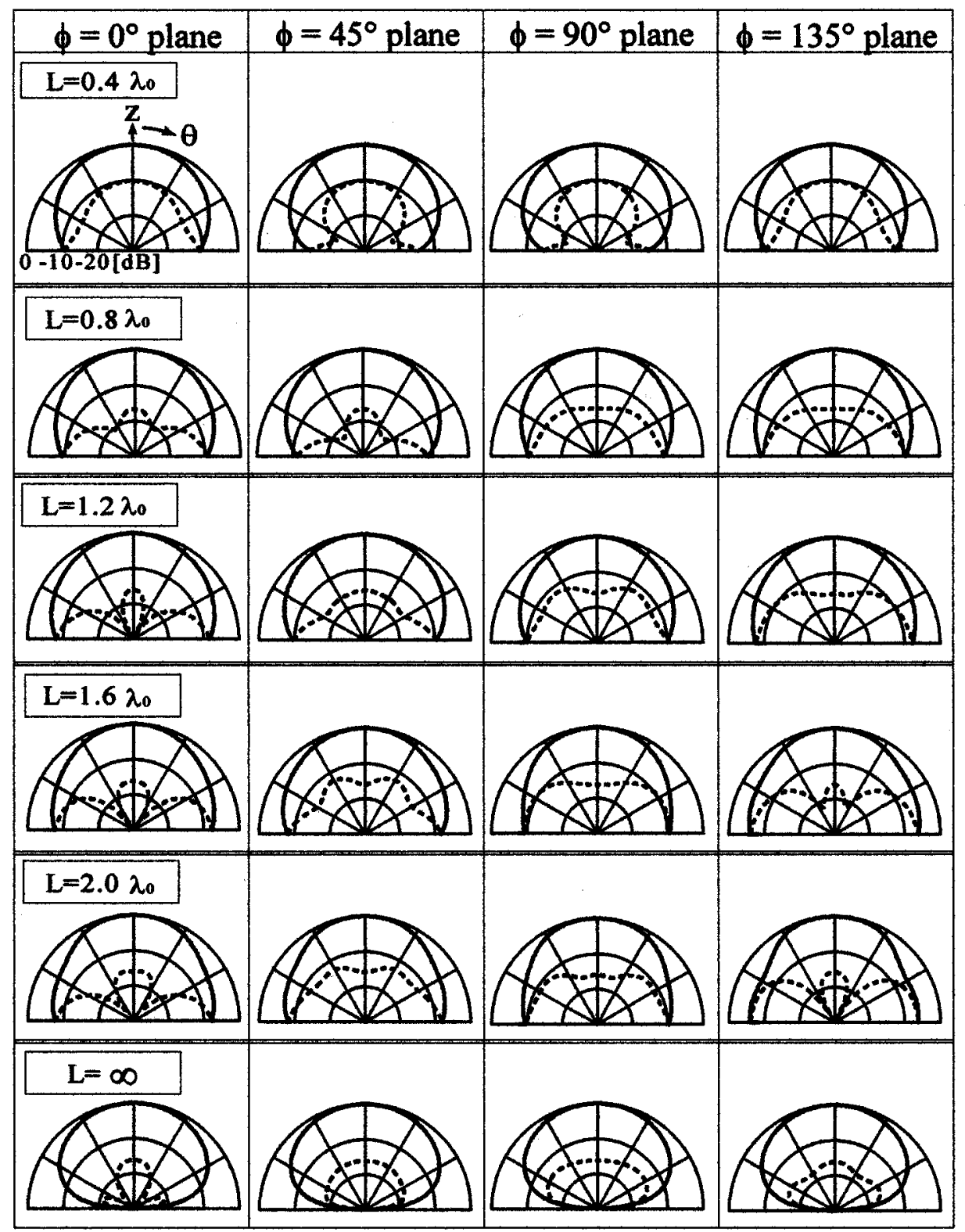

Fig. 7. Radiation patterns of $\mathrm{SA}_{\infty}$ as a function of side length $L$. Right-hand circularly polarized wave component $\mathrm{E}_{R}$-. Left-hand circularly polarized wave component $E_{L}$ - - -

where $P_{i n}$ is the power input to the antenna, i.e., $P_{i n}=$ Real $\left(V_{e} I_{e}^{*}\right)$, where $V_{e}$ and $I_{e}^{*}$ are the voltage and the complex conjugate of the current at the input terminals, respectively. $E_{R}$ is the intensity of an RHCP wave: $E_{R}=\left(E_{\theta}+j E_{\phi}\right) / 2$.

The axial ratio is given as $A R=\left(\left|E_{R}\right|+\left|E_{L}\right|\right) /\left(\left|E_{R}\right|\right.$ $\left.-\left|E_{L}\right|\right)$, where $E_{L}$ is the intensity of a left-hand circularly polarized (LHCP) wave: $E_{L}=\left(E_{\theta}-j E_{\phi}\right) / 2$.

\section{NUMERICAL RESULTS AND DISCUSSIONS}

It was confirmed that the FDTD computer programs developed on the basis of the theory in Section III reproduced the data shown in [16, Fig. 3]. After this validation, the following radiation characteristics of the spiral antennas printed on a finite-size dielectric substrate are obtained using the same computer programs.
A. Spiral Antenna on a Finite-Size Dielectric Substrate Backed by a Conducting Plane of Infinite Extent: $S A_{\infty}$

The first analysis is devoted to a spiral antenna on a finite-size dielectric substrate backed by a conducting plane of infinite extent, shown in Fig. 1(a). The configuration parameters are described in Section II.

Fig. 5 shows the current distribution as a function of dielectric substrate side length $L$. The current distributions along the two spiral arms are symmetrical with respect to the feed point $s_{f}$, and hence half of each current distribution is shown in this figure. The phase of the current distribution reveals that as $L$ increases, the currents along the arms tend toward a smoothly decaying traveling-wave. The current distribution for $L \geq 0.8 \lambda_{0}$ is almost the same as that for $L=\infty$. The guide wavelength of the current for $L=\infty$ is evaluated to be approximately 
$\lambda_{g}=0.78 \lambda_{0}$, which is close to a rough estimation of the guide wavelength, $\lambda_{\text {rough }}=\lambda_{0} / \varepsilon_{e f f}^{1 / 2}=0.775 \lambda_{0}$, where $\varepsilon_{e f f}$ is the average relative permittivity given as $\varepsilon_{e f f}=\left(1+\varepsilon_{r}\right) / 2$.

For comparison, the current distribution for $L=\infty$ obtained using the method of moments (MoM) [2] is also presented in Fig. 5(e). It is seen that the results shown in Fig. 5(d) agree well with those in Fig. 5(e). The good agreement again ensures that the developed FDTD computer programs correctly work. Note that the MoM in [2] can handle only the case where the dielectric is of infinite extent $(L=\infty)$.

Fig. 6 depicts the input impedance $Z_{i n}=R_{i n}+j X_{i n}$ as a function of substrate side length $L$. No significant variations are found for $L \geq 0.8 \lambda_{0}$. It is clear that the input impedance obtained by the FDTD method for $L=\infty$ is in good agreement with MoM analysis values, which are shown on the right side of Fig. 6.

The infinite conducting plane makes radiation unidirectional. The radiation patterns are shown in Fig. 7, where the far-field is decomposed into two wave components; right- and left-hand circularly polarized wave components. The left-hand circularly polarized wave component $\left[E_{L}=\left(E_{\theta}-j E_{\phi}\right) / 2\right.$, the cross polarization component], which degrades the axial ratio (AR) of a circular polarization antenna, is generated from the backward currents flowing toward the feed point. From the investigation of the current distributions shown in Fig. 5, it can be expected that the AR will improve as the substrate side length $L$ increases. This is confirmed by Fig. 8 .

Fig. 8 also shows the gain $G_{R}$ for an RHCP wave. The variation in the gain is not large (less than $1 \mathrm{~dB}$ ). The gain at $L=$ $0.8 \lambda_{0}$ is approximately $6.8 \mathrm{~dB}$. The gain for $L=\infty$ is in good agreement with that obtained using the MoM. The radiation efficiency $\eta_{\mathrm{rad}}$ for the $\mathrm{SA}_{\infty}$ with a substrate (and a conducting plane) of infinite extent is degraded due to surface wave effects. The $\eta_{\mathrm{rad}}$, which is calculated using the MoM, is $72 \%$. This corresponds to a loss of $1.4 \mathrm{~dB}$ in the gain.

Further analysis is performed to investigate the frequency response for $L=0.8 \lambda_{0}$. The frequency bandwidth for a $3-\mathrm{dB}$ axial ratio criterion is calculated to be $18.4 \%$. Over this bandwidth, the gain shows an almost constant value of $7 \mathrm{~dB}$ and the VSWR relative to a resistance value at a test frequency $f_{0}=8$ $\mathrm{GHz}(240 \Omega)$ is less than 2 , as desired.

\section{B. Spiral Antenna on a Finite-Size Dielectric Substrate Backed by a Finite-Size Conducting Plane: $S A_{\text {finite }}$}

The $\mathrm{SA}_{\text {finite }}$ shown in Fig. 1(b) has a compact structure and is suitable for practical applications. In this section, the effects of the finite side length of the square dielectric and conducting plane on the radiation characteristics are investigated using the configuration parameters described in Section II.

When the side length $L$ is changed, the current distribution exhibits almost the same behavior as that shown in Fig. 5. That is, as $L$ increases, the currents along the arms tend toward a smoothly decaying traveling-wave. This behavior of the current results in an input impedance characteristic similar to that shown in Fig. 6. The input impedance of the $\mathrm{SA}_{\text {finite, }}$, shown in Fig. 9, is almost the same as that of the $\mathrm{SA}_{\infty}$, provided that the side length $L$ is longer than $0.8 \lambda_{0}$.

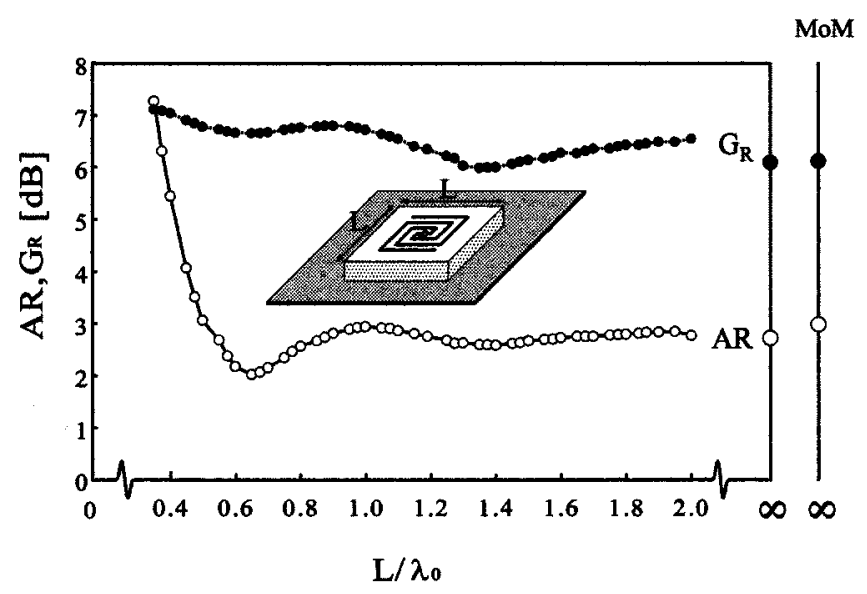

Fig. 8. Axial ratio AR and gain $G_{R}$ of $\mathrm{SA}_{\infty}$ as a function of normalized side length $\left(L / \lambda_{0}\right)$. Results calculated using MoM are also presented for $L=\infty$.

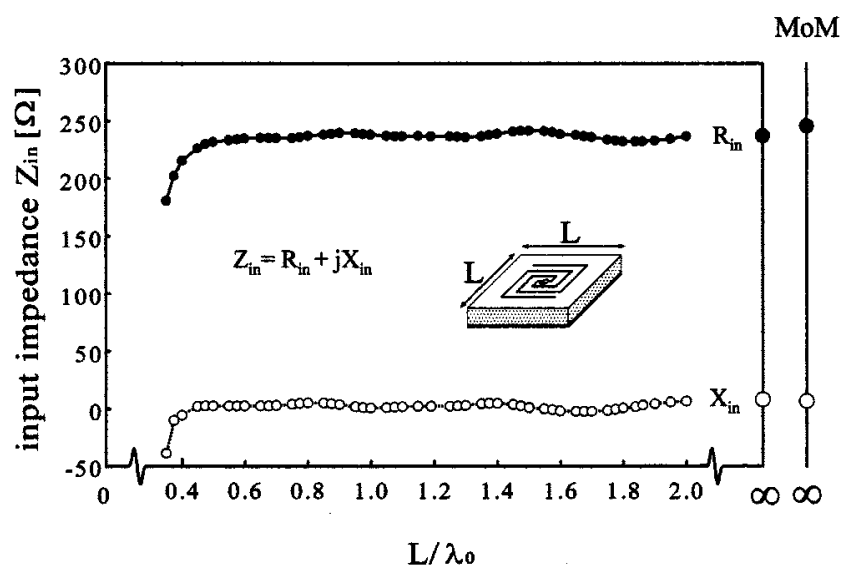

Fig. 9. Input impedance $Z_{i n}=R_{i n}+j X_{i n}$ of $\mathrm{SA}_{\text {finite }}$ as a function of normalized side length $\left(L / \lambda_{0}\right)$. Results calculated using MoM are also presented for $L=\infty$.

The radiation pattern of the $\mathrm{SA}_{\text {finite }}$, however, is different from that of the $\mathrm{SA}_{\infty}$. Fig. 10 shows the radiation pattern of the $\mathrm{SA}_{\text {finite }}$ as a function of the side length $L$. Due to the finite-size conducting plane, backlobes appear. Fig. 11 shows the HPBW of the main beam radiating a right-hand circularly polarized wave $E_{R}$. It is found that the HPBW becomes wider in the vicinity of $L / \lambda_{0}=1.3$. As a result, the gains in the vicinity of $L / \lambda_{0}=1.3$ are lower, as shown in Fig. 12. Note that the radiation from the $\mathrm{SA}_{\text {finite }}$ is circularly polarized in the vicinity of $L / \lambda_{0}=1.3$ with axial ratios of less than $3 \mathrm{~dB}$.

Further analysis is performed to evaluate the frequency response for the $\mathrm{SA}_{\text {finite. }}$. The frequency response is obtained for a dielectric substrate side length $L=0.8 \lambda_{0}$, which is the same as the side length used for investigating the frequency response for the $\mathrm{SA}_{\infty}$. The analysis reveals that the frequency bandwidth for a $3-\mathrm{dB}$ axial ratio criterion is $18.3 \%$, which is almost the same as that for the $\mathrm{SA}_{\infty}$. Over this bandwidth, the $\mathrm{SA}_{\text {finite }}$ has almost the same gain $(7 \mathrm{~dB})$ as the $\mathrm{SA}_{\infty}$ and the VSWR relative to a resistance value of the input impedance at a test frequency $f_{0}=8 \mathrm{GHz}(236 \Omega)$ is less than 2 . 


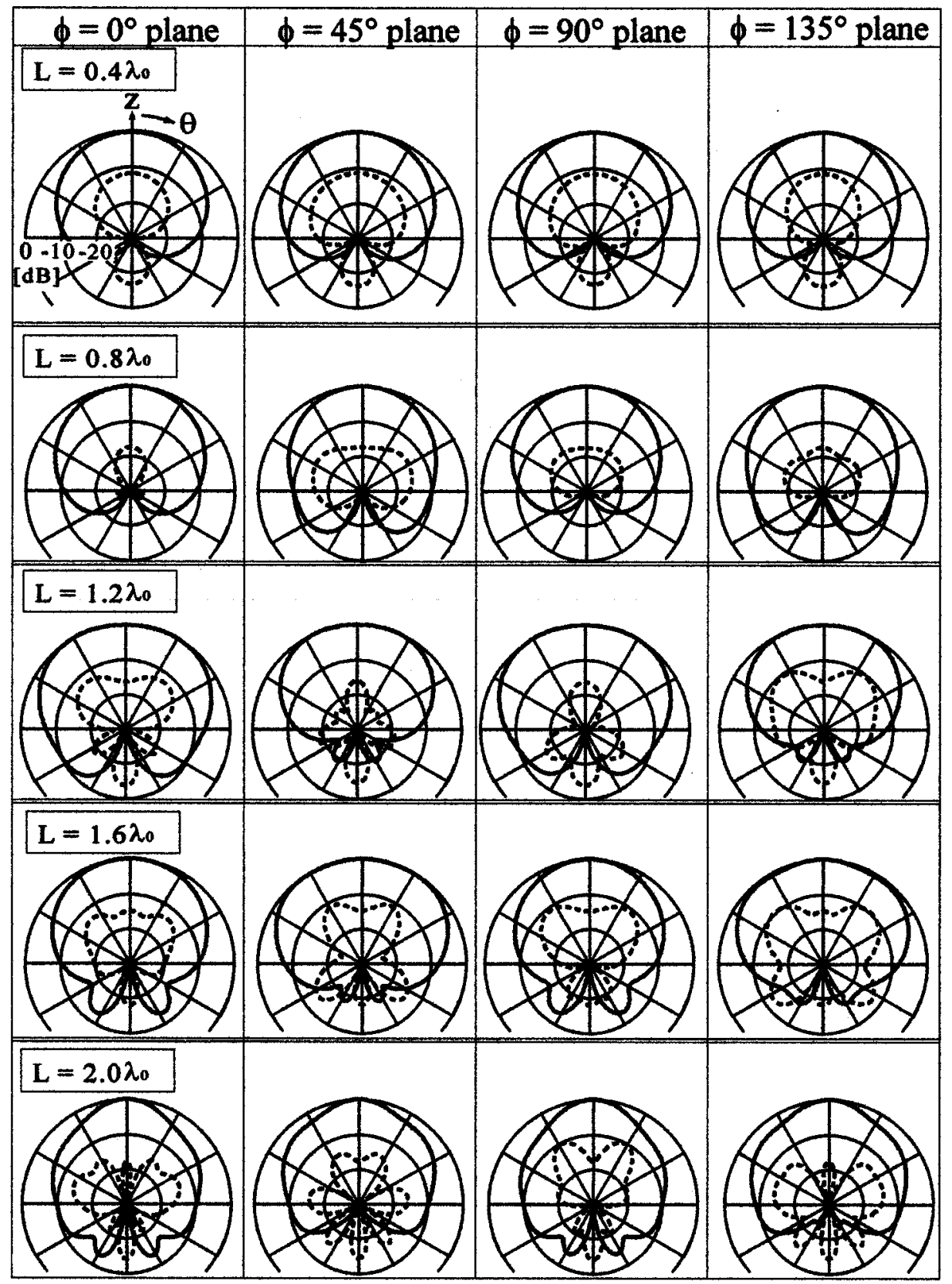

Fig. 10. Radiation patterns of $\mathrm{SA}_{\text {finite }}$ as a function of side length $L$. Right-hand circularly polarized wave component $E_{R}-$. Left-hand circularly polarized wave component $E_{L}--$.

\section{CONCLUSIONS}

The FDTD method has been used for analysis of printed spiral antennas. The computation space is reduced to one-half of the full computation space, making use of the symmetry of the spiral arms with respect to the feed point. The antenna characteristics are evaluated on the basis of the electric and magnetic fields obtained by the FDTD method.

The effects of a square dielectric substrate (of side length $L$ ) on the radiation characteristics have been revealed for two cases: 1) the conducting plane that backs the finite-size dielectric substrate is of infinite extent and 2) the conducting plane and dielectric substrate have the same finite-size (both are square, having the side length $L$ ). The spiral antennas for the first and second cases are designated as the $\mathrm{SA}_{\infty}$ and $\mathrm{SA}_{\text {finite }}$, respectively.

As the side length $L$ increases, the currents along the arms tend toward a smoothly decaying traveling-wave in both $\mathrm{SA}_{\infty}$ and $\mathrm{SA}_{\text {finite. The input impedance, therefore, becomes constant }}$ at a certain value of $L$ ( $L \geq 0.8$ wavelength). The $\mathrm{SA}_{\infty}$ radiates a unidirectional beam (without back-lobes) due to the conducting plane of infinite extent, while the $\mathrm{SA}_{\text {finite }}$ has backlobes due to the finite-size conducting plane. The difference between the radiation patterns of the $\mathrm{SA}_{\infty}$ and $\mathrm{SA}_{\text {finite }}$ is related to differences in the axial ratio and gain characteristics of these antennas. The variations in the gain and axial ratio of the $\mathrm{SA}_{\infty}$ are relatively small compared with those of the $\mathrm{SA}_{\text {finite }}$. Analysis has also revealed that when the $\mathrm{SA}_{\infty}$ and the $\mathrm{SA}_{\text {finite }}$ have 


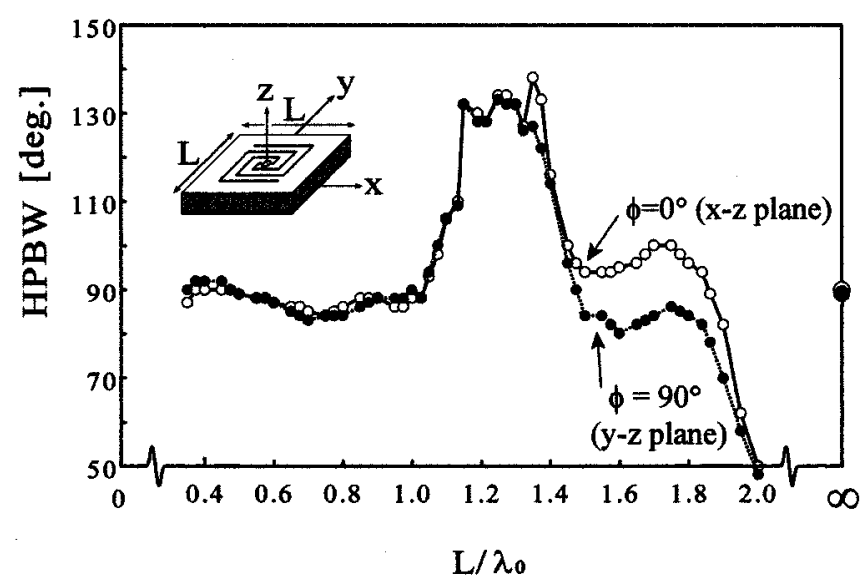

Fig. 11. Half-power beam width (HPBW) of $\mathrm{SA}_{\text {finite }}$ as a function of normalized side length $\left(L / \lambda_{0}\right) \cdot \phi=0^{\circ}$ plane $(x-z$ plane $) \circ \circ \circ \circ \quad \phi=90^{\circ}$ plane $(y-z$ plane $) \bullet \bullet \bullet \bullet$.

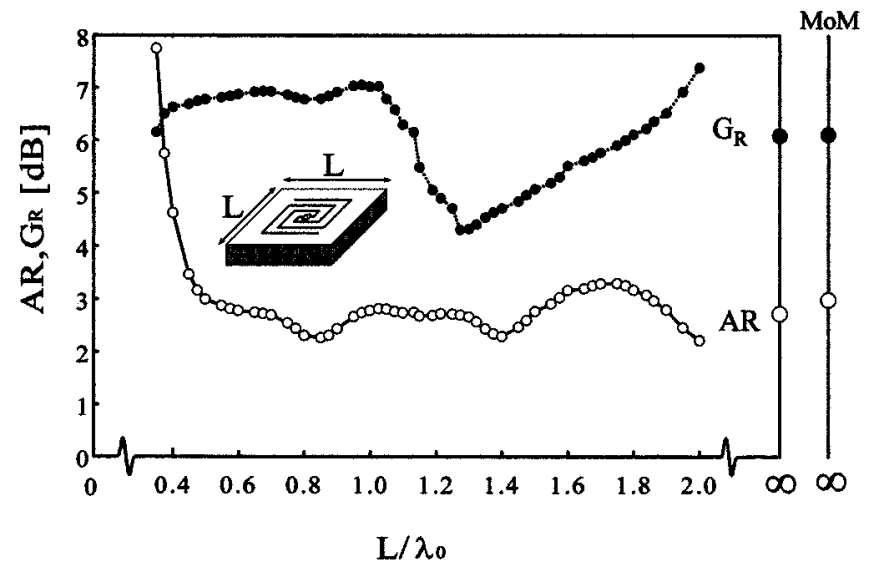

Fig. 12. Axial ratio $\mathrm{AR}$ and gain $G_{R}$ of $\mathrm{SA}_{\text {finite }}$ as a function of normalized side length $\left(L / \lambda_{0}\right)$. Results calculated using MoM are also presented for $L=$ $\infty$

the same side length $L=0.8 \lambda_{0}$, they show almost the same frequency bandwidth (approximately 18\%) for a $3-\mathrm{dB}$ axial ratio criterion and have a gain of approximately $7 \mathrm{~dB}$.

\section{ACKNOWLEDGMENT}

The authors would like to thank V. Shkawrytko for his kind assistance in the preparation of this manuscript.

\section{REFERENCES}

[1] H. Nakano, K. Nogami, S. Arai, H. Mimaki, and J. Yamauchi, "A spiral antenna backed by a conducting plane reflector," IEEE Trans. Antennas Propagat., vol. AP-34, pp. 791-796, June 1986.

[2] H. Nakano, S. R. Kerner, and N. G. Alexopoulos, "The moment method solution for printed wire antennas of arbitrary configuration," IEEE Trans. Antennas Propagat., vol. 36, pp. 1667-1674, Dec. 1988.

[3] J. J. H. Wang and V. K. Tripp, "Design of multioctave spiral-mode microstrip antennas," IEEE Trans. Antennas Propagat., vol. 39, pp. 332-335, Mar. 1991.

[4] L. Shafai, "Design of multi-arm multi-mode spiral antennas for directional beams using equivalent array concept," Electromagn., vol. 14, no. 3-4, pp. 285-304, July-Dec. 1994.

[5] N. J. Champagne, II., J. T. Williams, and D. R. Wilton, "Analysis of resistively loaded, printed spiral antennas," Electromagn., vol. 14, no. 3-4, pp. 363-395, July-Dec. 1994.
[6] P. E. Mayes, "Planar and other wide-angle logarithmic spirals over ground," Electromagn., vol. 14, no. 3-4, pp. 329-362, July-Dec. 1994.

[7] U. R. Kraft, "Polarization properties of small printed spiral antennas with four resistively loaded arms," IEE Proc. Microw. Antennas Propag., vol. 144, pp. 131-135, April 1997.

[8] T. Ozdemir, M. W. Nurnberger, and J. Volakis, "A thin cavity-backed Archimedean slot spiral for $800-3000 \mathrm{MHz}$ band coverage," in IEEE Antennas Propagat. Int. Symp. Dig., vol. 4, June 1998, pp. 2336-2339.

[9] S. K. Khamas, G. G. Cook, R. J. Waldron, and R. M. Edwards, "Moment method analysis of printed single-arm wire spiral antennas using curved segments," IEE Proc. Microw. Antennas Propagat., vol. 144, pp. 261-265, Aug. 1997.

[10] H. Nakano, H. Yasui, and J. Yamauchi, "A square spiral antenna," in Proc. 1997 IEICE General Conf., Osaka, Japan, Mar. 1997, pp. B-1-B-124.

[11] A. Taflove, Computational Electrodynamics. Norwood, MA: Artech House, 1995.

[12] T. Uno, "Field and antenna analysis by FDTD method," Institute of Electronics, Information and Communications Engineers in Japan, Tutorial Text, Mar. 1996.

[13] K. S. Yee, "Numerical solution of initial boundary value problems involving Maxwell's equations in isotropic media," IEEE Trans. Antennas Propagat., vol. AP-14, pp. 302-307, May 1966.

[14] Z. P. Liao, H. L. Wong, B. P. Yang, and Y. F. Yuan, "A transmitting boundary for transient wave analysis," Sci. Sinica, ser. A, vol. 27, no. 10, pp. 1063-1076, 1984

[15] H. Nakano, J. Eto, Y. Okabe, and J. Yamauchi, "Tilted- and axial-beam formation by a single-arm rectangular spiral antenna with compact dielectric and conducting plane," IEEE Trans. Antennas Propagat., vol. 49, pp. 17-24, Dec. 2001.

[16] H. Nakano, K. Hirose, T. Suzuki, S. R. Kerner, and N. G. Alexopoulos, "Numerical analyzes of printed line antennas," IEE Proc. Pt. H, vol. 136, pp. 98-104, Apr. 1989.

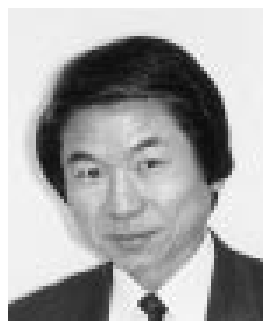

Hisamatsu Nakano (M'75-SM'87-F'92) was born in Ibaraki, Japan. He received the B.E., M.E., and Dr.E. degrees in electrical engineering from Hosei University, Tokyo, Japan, in 1968, 1970, and 1974, respectively.

Since 1973, he has been a Member of the Faculty of Hosei University, where he is now a Professor of electronic informatics. His research topics include numerical methods for antennas, electromagnetic wave scattering problems, and light wave problems. He has published more than 170 refereed journal papers and 130 international symposium papers on antenna and relevant problems. He is the author of Helical and Spiral Antennas (New York: Research Studies Press, 1987). He published the chapter "Antenna analysis using integral equations," in Analysis Methods of Electromagnetic Wave Problems, vol. 2 (Norwood, MA: Artech House, 1996). He was a Visiting Associate Professor at Syracuse University, Syracuse, NY, from March to September 1981, a Visiting Professor at the University of Manitoba, Canada, from March to September 1986, and a Visiting Professor at the University of California, Los Angeles, from September 1986 to March 1987.

Dr. Nakano received an International Scientific Exchange Award from the Natural Sciences and Engineering Research Council of Canada in 1986. In 1987, he received the Best Paper Award from the IEE 5th International Conference on Antennas and Propagation. In 1994, he received the IEEE AP-S Best Application Paper Award (H. A. Wheeler Award). He is an Associate Editor of IEEE Antennas and Propagation Magazine and a member of the AP-S administrative committee.

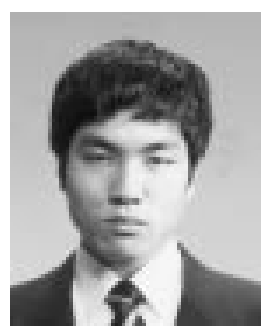

Hajime Yasui received the B.E. and M.E. degrees from Hosei University, Tokyo, Japan, in 1997 and 1999 , respectively.

He joined The Tokyo Metropolitan Government, Tokyo, Japan, in 1999.

Mr. Yasui is a member of the Institute of Electronics, Information and Communication Engineers (IEICE) of Japan. 


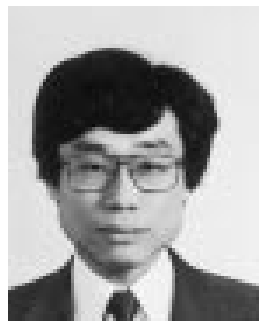

Junji Yamauchi (M'85) was born in Nagoya, Japan, on August 23, 1953. He received the B.E., M.E., and Dr.E. degrees from Hosei University, Tokyo, Japan, in 1976, 1978, and 1982, respectively.

From 1984 to 1988, he served as a Lecturer in the Electrical Engineering Department, Tokyo Metropolitan Technical College, Tokyo. Since 1988, he has been a Member of the Faculty of Hosei University, where he is now a Professor of electronic informatics. His research interests include optical waveguides and circularly polarized antennas. $\mathrm{He}$ is the author of Propagating Beam Analysis of Optical Waveguides (U.K.: Research Studies Press, 2002).

Dr. Yamauchi is a member of the Optical Society of America (OSA) and the Institute of Electronics, Information and Communication Engineers (IEICE) of Japan. 